\title{
Hydrocarbon film deposition inside cavity samples in remote areas of the JET divertor during the 1999-2001 and 2005-2009 campaigns
}

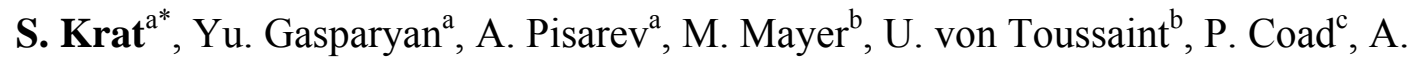 \\ Widdowson $^{\mathrm{c}}$, JET-EFDA contributors $^{1}$ \\ ${ }^{a}$ National Research Nuclear University MEPhI (Moscow Engineering Physics Institute), Moscow \\ Kashirskoe road 31, 115409, Russia;. \\ ${ }^{b}$ Max-Planck-Institut für Plasmaphysik, Boltzmannstr. 2, 85748 Garching, Germany. \\ ${ }^{c} J E T-E F D A$, Culham Science Centre, Abingdon OX14 3DB, UK
}

${ }^{1}$ See the Appendix of F. Romanelli et al., Proceedings of the 24th IAEA Fusion Energy Conference 2012, San Diego, USA

\begin{abstract}
Hydrocarbon film deposition was studied with cavity samples in remote areas of the inner and outer JET divertor and below the divertor septum during the 1999-2001 and 20052009 campaigns. Thick hydrocarbon films were formed inside the cavities. These deposited hydrocarbon layers have high $\mathrm{D} / \mathrm{C}$ ratios close to 1 . The formation of these films is mainly due to sticking of hydrocarbon particles with high surface loss probabilities $>0.6$. The observed surface loss probabilities depend on the position in the divertor and vary during different campaigns. The particles responsible for hydrocarbon layer formation originate from the divertor strike points. Except for the septum cavity the deposition of beryllium was very low and showed a very different distribution from that of deuterium and carbon.
\end{abstract}

PACS: 68.55.jd, 52.55.Fa, 61.05.Np 
PSI-21 keywords: Deuterium, Carbon films, co-deposition, JET

Corresponding Author Address: 115409, Russia, Moscow, Kashirskoe shosse 31

Corresponding Author e-mail: stepan.krat@gmail.com

Presenting Author: Stepan Krat

Presenting Author e-mail: stepan.krat@gmail.com 


\section{Introduction}

Redeposition of material eroded from plasma-facing components in remote areas of fusion devices can lead to accumulation of hydrogen isotopes (including radioactive tritium) in codeposited layers with carbon and beryllium [1-2]. Hydrocarbon film formation has been observed in various experiments [3-6], including "full metal" ones [7] in remote regions, such as shadowed areas of the divertor, only accessible by neutral particles. It has been already speculated, that hydrocarbon film formation might be due to sticking of hydrocarbon radicals with sticking coefficients below 1, i.e. radicals capable of surviving several wall collisions before finally sticking to the wall and forming a hydrocarbon layer [8]. It has been shown already by time-resolved quarz micro-balances in the JET divertor [9], that thermal decomposition of hydrocarbon layers on divertor tiles by ELMs ejects radicals or small clusters, which then can form layers in shadowed areas. However, the properties of these ejected particles, especially their sticking coefficients, remain still not understood.

In 2010 the first wall of JET has been changed from carbon to the ITER-like Be-W configuration [10]. In preparation for data from the new experimental campaigns, results obtained during different campaigns of the carbon phase of JET operation should be analyzed, so that they can be then compared with new results [11-12], thus elucidating differences between the transport of carbon and of metals.

Hydrocarbon radicals hitting a wall may adsorb with a sticking probability $s$, can react to a non-reactive molecule with probability $\gamma$, or can be reflected with probability $r$. The surface loss probability $\beta$ is given by $\beta=s+\gamma$, with $r+s+\gamma=1$. In this paper, a comparative analysis of hydrocarbon film sources and surface loss probabilities in remote areas of the divertor of JET during the 1999-2001 and 2005-2009 campaigns is presented. 


\section{Experimental}

\subsection{Cavity samples}

Cavity samples were used to measure film deposition. They consist of two parallel silicon plates in a metal frame, forming a cavity. The top plate has an entrance slit of a known width $(0.8 \mathrm{~mm})$. The dimensions of the cavity are $18.6 \times 15 \times 2.2 \mathrm{~mm}$, with the slit in the middle of the longer side of the plate. Hydrocarbon radicals entering the cavity through the slit can either stick to the bottom plate and form a precursor for film deposition, they can be reflected from it, or they can transform into a non-reactive molecule. This forms a film thickness distribution from which information about the sticking coefficient can be acquired. If the sticking coefficient is high, then the probability for sticking at the first hit surface is large: In that case the cavities act as pinhole cameras which allow to reconstruct the source distribution of the radicals.

Two cavity samples were placed in the divertor of JET during the 1999-2001 campaign (Fig. 1a). One was located close to the louvers of the inner divertor, the other was located under the divertor septum. During this time 6949 successful discharges with $9.69 \times 10^{4}$ $\mathrm{s}$ total divertor plasma time were performed.

Two cavity samples were successfully retrieved from the divertor of JET after the discharge campaign of 2005-2009 (Fig. 1b). One was located close to the louvers of the inner divertor at the same place as the inner divertor cavity used during the 1999-2001 campaign. Another was located close to the louvers of the outer divertor. During this time 12042 successful discharges with $23.6 \times 10^{4} \mathrm{~s}$ total divertor plasma time were performed.

The cavity slits were oriented horizontal in toroidal direction facing the divertor plasma (see Fig. 1). 


\subsection{Ion beam analysis}

Deposited layers were quantitatively analyzed using nuclear reaction analysis (NRA). Deuterium was detected using the $\mathrm{D}\left({ }^{3} \mathrm{He}, \mathrm{p}\right)^{4} \mathrm{He}[13]$ nuclear reaction at 2500, 3500, 4500 and $6000 \mathrm{keV}$ incident energies. For 2005-2009 samples carbon was detected using the ${ }^{12} \mathrm{C}\left({ }^{3} \mathrm{He}, \mathrm{p} 0\right){ }^{14} \mathrm{~N},{ }^{12} \mathrm{C}\left({ }^{3} \mathrm{He}, \mathrm{p} 1\right){ }^{14} \mathrm{~N}$ and ${ }^{12} \mathrm{C}\left({ }^{3} \mathrm{He}, \mathrm{p} 2\right){ }^{14} \mathrm{~N}$ nuclear reactions [14] at the same

energies, beryllium was detected using ${ }^{9} \mathrm{Be}\left({ }^{3} \mathrm{He}, \mathrm{p} 0\right){ }^{11} \mathrm{~B}$ and ${ }^{9} \mathrm{Be}\left({ }^{3} \mathrm{He}, \mathrm{p} 1\right){ }^{11} \mathrm{~B}$ reactions [15]. For 1999-2001 samples, carbon and beryllium content was detected using Rutherford backscattering method with $1.5 \mathrm{MeV}$ protons. The NRA detector was covered with $5 \mu \mathrm{m} \mathrm{Ni}$ and $12 \mu \mathrm{m}$ Mylar foils to stop backscattered ${ }^{3} \mathrm{He}$ ions. The spectra were analyzed using the SIMNRA program [16].

\subsection{Computer simulation}

Computer simulations of particle deposition inside the cavities were made using a Monte-Carlo simulation[17]. A 2-dimensional model was used, simulating the profile in the central plane of the cavity, perpendicular to the slit. Generally, a statistic of $2 \times 10^{8}$ incident particles was used for each modeled profile.

At each surface the particles could stick with probability $\mathrm{s}$, reflect with probability $\mathrm{r}$, or transform into a stable molecule with the probability $\gamma$. The surface loss probability is $\beta=\mathrm{s}+\gamma$, with $\beta+\mathrm{r}=1 . \gamma=0$ was used in all simulations.

The incident particle flux was separated into 20 sub-fluxes. Each sub-flux consisted of particles entering the cavity with a random angle of incidence in a small range. The sum of all sub-flux ranges comprised the whole range of directions particles could enter the cavity from. The ranges didn't overlap. The resulting particle flux was a linear combination of all sub- 
fluxes. The particle flux was reconstructed by fitting the deposition profile on the bottom plate of the cavity sample using the sticking coefficient of $s=1$ and varying the configuration of the incoming particle flux.

Particles are reflected with the $\cos ^{\alpha}(\theta)$ distribution from all surfaces, where $\alpha$ is typically 1 in accordance with the experimental data [8], but can be adjusted to achieve a better fit.

The neutral gas pressure in the divertor is typically below $10^{-1} \mathrm{~Pa}$ [18] The gas pressure inside the cavities was therefore assumed to be $<10^{-1} \mathrm{~Pa}$ which results in a mean free path length $>8 \mathrm{~mm}$. This allowed collisions with gas molecules in the cavity to be neglected.

The resulting deposition profile was calculated as the weighted sum of the deposition profiles calculated for different sticking coefficients. The sticking coefficients modeled were $[0.001,0.01 . .0 .09,0.1 . .0 .9,0.91-099,1]$. The modeled profiles were normalized to the same total amount of particles deposited on both top and bottom plates of the cavity samples. The surface loss probability was obtained by matching experimental and modeled deposition profiles. The fitting was done in the linear scale. Additionally, it was estimated by comparing the experimental and modeled ratios of the total amount of particles deposited on the bottom plate to the total amount of particles deposited on the upper plate.

3. Results

\subsection{Inner divertor}

The incident particle flux distribution for the 1999-2001 and 2005-2009 campaigns at the inner divertor sample, as reconstructed from the deposition of $\mathrm{D}$ at the bottom plate, is shown in Fig. 2. In both campaigns most of the particles originated from the direction about 
$20^{\circ}$ below the horizontal direction, which corresponds to the sloped central area of divertor tile 4. The distribution in 2005-2009 was more peaked than in 1999-2001. The sloped central area of tile 4 contained very thick codeposited hydrocarbon layers, and the inner divertor strike point was located there regularly. A second considerably smaller peak in the incident particle flux distribution points towards a position located $25^{\circ}$ above the horizontal direction towards the rear side of tile 3 .

Experimentally measured profiles for carbon, deuterium and beryllium deposition inside the inner divertor cavities during the 1999-2001 and 2005-2009 campaigns are shown in figures $3 \mathrm{a}$ and $3 \mathrm{~b}$, respectively. The layers in the center area with a width of about $2 \mathrm{~mm}$ of the bottom plate of the 2005-2009 sample were thicker than $4.5 \times 10^{24}(\mathrm{D}+\mathrm{C})$ atoms $/ \mathrm{m}^{2}$, which precluded full inventory of deuterium atoms there. The profiles for carbon and deuterium generally had the same shape, and the films had a high $\mathrm{D} / \mathrm{C}$ ratio close to unity.

The beryllium thickness distribution was almost homogeneous on the top and bottom plates, with an average thickness of about $1 \times 10^{21}$ atoms $/ \mathrm{m}^{2}$. The beryllium signal in the area of the bottom plate opposite the entrance slit was smaller than the background signal, so that beryllium could not be detected here. Due to the overlapping with signals from other nuclear reactions the beryllium content was smaller than about $2 \%$ in this area.

The sticking coefficient of hydrocarbon radicals was derived using the deuterium distribution in the cavities, see Fig. 3. For the 1999-2001 cavity the surface loss probability was $\beta=0.95$ according to the deuterium profile at the bottom and top plates, and $0.92<\beta<0.95$ taking only the ratios of D-atoms on the bottom of the cavity to the amount of D-atoms on the top plate of the cavity into account. For the 2005-2009 campaign sample, the obtained surface loss probabilities using the same methods were $\beta=0.76$ and $0.7<\beta<0.8$, respectively. 
The deposition patterns obtained using the $\cos (\vartheta)$ distribution as angular distribution for particles reflected from the walls of the cavities doesn't provide a very good match for the particle distribution on the upper plates. The experimentally measured distribution was steeper with higher thicknesses near the entrance slit and lower ones in the outer areas. The deuterium deposition profile corresponds best with an over-cosine distribution for reflected particles with $\cos ^{\alpha}(\vartheta)$, where $\alpha=4$ (see Fig. 3). With this distribution, $\beta$ obtained using profilematching was 0.94 for $1999-2001$ sample and remained 0.76 for $2005-2009$ samples; it is quite robust with respect to changes in $\alpha$.

The homogeneous distribution of beryllium could be modelled with a very low sticking coefficient. However, as this seems rather unphysical and the beryllium deposition is low compared to deuterium and carbon, we assume that the homogeneous beryllium deposition is due to a contamination by beryllium containing dust during vent of the machine or during handling in the beryllium-handling facility.

\subsection{Septum cavity}

The source of particles comprising the film in the septum cavity sample from the 1999-2001 campaign (fig. 4) was highly peaked, with an overwhelming amount of particles originating from a finite area in the center of divertor tile 3, near the strike point location.

Experimentally measured profiles for $\mathrm{C}, \mathrm{D}$ and $\mathrm{Be}$, as well as modeling results for the deposition inside the cavity are shown in figure 5. The determined surface loss probability was $\beta=0.98$, according to both analysis methods (i.e. fitting the whole distribution and the ratio of top to bottom deposition, see the previous section). The fit could be improved by adding $0.5 \%$ of low-sticking $(\beta \approx 0.001)$ hydrocarbon species into the model. Accounting for the probability of particles escaping from the cavity, this corresponds to a 1:4 ratio of high- 
sticking to low-sticking species particle fluxes. This shows that the contribution of lowsticking particles to the divertor deposition is very low, although the fluxes of low-sticking particles can be high. $\operatorname{Cos}(\vartheta)$ distribution for the particles reflected from the walls of the cavities agrees well with the experimental data, while the $\cos ^{4}(\vartheta)$ distribution gives a worse fit, unlike for the other cavities.

The beryllium thickness distribution roughly corresponds with the $\mathrm{C}$ and $\mathrm{D}$ distributions, at least on the upper plate of the cavity, and had a maximum thickness of about $1 \times 10^{22}$ atoms $/ \mathrm{m}^{2}$.

\subsection{Outer divertor cavity}

Experimentally measured deposition profiles for $\mathrm{C}, \mathrm{D}$ and $\mathrm{Be}$, as well as modeling results are shown in figure 6 . The deposited film in the center of the bottom plate of the outer divertor cavity from the 2005-2009 campaign peeled off prior to the measurement. This made the reconstruction of the particle flux distribution impossible. For modeling purposes, the flux distribution for the inner divertor cavity of the 2005-2009 campaign was used, as it showed good agreement with the remaining film thickness distribution.

The surface loss probability was $0.6<\beta<0.7$. Due to the lack of data from the center of the bottom plate it wasn't possible to use the integral ratio matching method for the analysis of this cavity with good accuracy, but, applying it only to the data from the outer regions of the bottom plate and the upper plates, the closest match between experimental and modeled data was $0.6<\beta<0.7$.

The beryllium thickness distribution was almost homogeneous, with the average thickness of about $1-2 \times 10^{21}$ atoms $/ \mathrm{m}^{2}$. 
As with the inner divertor cavities, the $\cos ^{4}(\vartheta)$ distribution for the particles reflected from the surfaces of the cavity provided a better agreement between experimental and modeled data than the $\cos (\vartheta)$ distribution did. B obtained for such distribution was $\beta \approx 0.6$.

\section{Discussion}

Based on time-resolved measurements using quartz micro-balances in a location not too far away of the inner divertor cavity sample the following model for carbon transport to remote areas of the inner divertor was developed in [9]:

1. Hydrocarbon layers are deposited on the horizontal inner divertor tile 4, for example during operation with the inner strike point on vertical tile 3 .

2. With the inner divertor strike point on tile 4, these hydrocarbon layers are eroded during ELM impact. This erosion is strongly non-linear, and ELMs with large energy content exhibit a much larger erosion than smaller ELMs. The proposed erosion mechanism is thermal decomposition of the layers into hydrocarbon radicals or small clusters.

3. The ejected erosion products then stick to surfaces of remote areas, where subsequently thick hydrocarbon layers are formed.

The pinhole camera effect of our cavity samples allows to reconstruct the incident particles flux distribution, thus allowing to add spatial information to the above model. This spatial information confirms the above model: The particles responsible for film formation indeed originate from the plasma strike point. For the septum this is the strike point position on tile 3, while for the inner divertor louvers the strike point position on the sloping part of tile 4 is responsible for the deposition. 
The ejected particles have mainly surface loss probabilities in the range $0.6<=\beta<=0.98$, i.e. they don't stick necessarily to the first surface they hit. The thickest layers are formed in lineof-sight to the strike point position, but layers can be also formed without direct line of sight (such as on the top inner plates of the cavities). The observed sticking coefficients depend on position in the divertor and on campaign. This can indicate that the ejected particle species distribution depends on plasma parameters and can vary depending on discharge and machine history.

All films except the ones in the septum cavity sample from 1999-2001 campaign could be adequately modeled using just one species with relatively high surface loss probability. The used cavities have only a small sensitivity for particles with low sticking coefficient, and it is therefore difficult to derive conclusions about fluxes of low-sticking species. Species with very low sticking coefficient $(\beta<=0.01)$ do not contribute significantly to layer formation in the investigated divertor areas, although even large fluxes of low-sticking species cannot be excluded. Low-sticking species would become more visible in areas which can be reached only after several wall bumps, such as pump ducts. However, at least in ASDEX Upgrade only very little hydrocarbon deposition was observed there [19]

One possible explanation for why the distribution of particles on the upper plates of the cavities doesn't correspond well with a $\cos (\vartheta)$ distribution may be the resputtering of the particles from the central area of the bottom plate [20], caused by energetic particle bombardment. For all the films, a sharp decline could be observed in the outermost parts of the cavities. This may be caused by isotopic exchange occurring in the very thin hydrocarbon films in those areas, or film degradation due to contact with atmospheric air. This effect is more pronounced for the outer divertor cavity sample.

\section{Conclusions}


Measurements with cavity samples in remote areas of the inner and outer JET divertor and below the divertor septum during the 1999-2001 and 2005-2009 campaigns with full carbon walls show the formation of thick hydrocarbon films inside the cavities. These deposited hydrocarbon layers have high $\mathrm{D} / \mathrm{C}$ ratios close to 1 . The formation of these films is mainly due to sticking of hydrocarbon particles with high surface loss probabilities $\beta>0.6$. The observed surface loss probabilities depend on the position in the divertor and vary during different campaigns. Low sticking species with surface loss probabilities $\beta<0.01$ cannot be excluded, but they have only a very small contribution to the layer formation in the divertor. The particles responsible for hydrocarbon layer formation originate from the divertor strike points. This confirms the model presented in [9], that thermal decomposition of hydrocarbon layers on tiles 3 and 4 by ELM impact is responsible for the formation of hydrocarbon layers in remote areas.

Except for the septum cavity the deposition of beryllium was very low and showed a very different distribution than deuterium and carbon. This gives rise to the hope, that transport of beryllium to remote divertor areas and codeposition of hydrogen isotopes will decrease significantly with the ITER-like wall.

\section{Acknowledgements}

This work was supported by EURATOM and carried out within the framework of the European Fusion Development Agreement. The views and opinions expressed herein do not necessarily reflect those of the European Commission.

Work supported by Russian Ministry of Education and Science 
References

[1] M. Mayer, V. Philipps, P. Wienhold, H.G. Esser, J. von Seggern, M. Rubel, Hydrogen inventories in nuclear fusion devices, Journal of Nuclear Materials, 290-293 (2001) 381-388.

[2] G. Federici, J.N. Brooks, D.P. Coster, G. Janeschitz, A. Kukuskhin, A. Loarte, H.D. Pacher, J. Stober, C.H. Wu, Assessment of erosion and tritium codeposition in ITER-FEAT, Journal of Nuclear Materials, 290-293 (2001) 260-265.

[3] J.P. Coad, N. Bekris, J.D. Elder, S.K. Erents, D.E. Hole, K.D. Lawson, G.F. Matthews, R.D. Penzhorn, P.C. Stangeby, Erosion/deposition issues at JET, Journal of Nuclear Materials, 290-293 (2001) 224230.

[4] M. Yoshida, T. Tanabe, A. Adachi, T. Hayashi, T. Nakano, M. Fukumoto, J. Yagyu, Y. Miyo, K. Masaki, K. Itami, Carbon transport and fuel retention in JT-60U with high temperature operation based on postmortem analysis, Journal of Nuclear Materials, 438, Supplement (2013) S1261-S1265.

[5] J. Von Seggern, A. Kirschner, V. Philipps, P. Wienhold, Deposition of hydrogen rich carbon films in pump ducts of TEXTOR, in: Physica Scripta T, 2004, pp. 118-122.

[6] L. Begrambekov, V. Barsuk, M. Dubrov, A. Kaplevsky, N. Klimov, D. Kovalenko, A. Kuzmin, A. Mischenko, V. Podkovyrov, P. Shigin, A. Zhitlukhin, A. Zakharov, Deuterium trapping in carbon films formed in different deposition conditions, Journal of Nuclear Materials, 438 (2013) S971-S974.

[7] M. Mayer, V. Rohde, K. Sugiyama, J.L. Chen, X. Gong, C. Hopf, J. Likonen, S. Lindig, R. Neu, G. Ramos, E. Vainonen-Ahlgren, A. Wiltner, Carbon balance and deuterium inventory from a carbon dominated to a full tungsten ASDEX Upgrade, Journal of Nuclear Materials, 390-391 (2009) 538-543.

[8] A. von Keudell, C. Hopf, T. Schwarz-Selinger, W. Jacob, Surface loss probabilities of hydrocarbon radicals on amorphous hydrogenated carbon film surfaces: Consequences for the formation of redeposited layers in fusion experiments, Nucl. Fusion, 39 (1999) 1451-1462.

[9] A. Kreter, H.G. Esser, S. Brezinsek, J.P. Coad, A. Kirschner, W. Fundamenski, V. Philipps, R.A. Pitts, A. Widdowson, Nonlinear impact of edge localized modes on carbon erosion in the divertor of the JET tokamak, Physical Review Letters, 102 (2009).

[10] G.F. Matthews, M. Beurskens, S. Brezinsek, M. Groth, E. Joffrin, A. Loving, M. Kear, M.L. Mayoral, R. Neu, P. Prior, V. Riccardo, F. Rimini, M. Rubel, G. Sips, E. Villedieu, P.d. Vries, M.L. Watkins, E.-J. contributors, JET ITER-like wall-overview and experimental programme, Physica Scripta, T145 (2011) 014001.

[11] K. Heinola, C.F. Ayres, A. Baron-Wiechec, J.P. Coad, J. Likonen, G.F. Matthews, A. Widdowson, J.E. Contributors, Tile profiling analysis of samples from the JET ITER-like wall and carbon wall, Physica Scripta, T159 (2014) 014013.

[12] T. Loarer, S. Brezinsek, V. Philipps, J. Bucalossi, D. Douai, H.G. Esser, S. Grunhagen, J. Hobirk, S. Jachmich, E. Joffrin, U. Kruezi, C. Lowry, G. Matthews, R. Smith, E. Tsitrone, S. Vartanian, Comparison of long term fuel retention in JET between carbon and the ITER-Like Wall, Journal of Nuclear Materials, 438, Supplement (2013) S108-S113.

[13] V.K. Alimov, M. Mayer, J. Roth, Differential cross-section of the $D\left({ }^{3} \mathrm{He}, \mathrm{p}\right)^{4} \mathrm{He}$ nuclear reaction and depth profiling of deuterium up to large depths, Nuclear Instruments and Methods in Physics Research Section B: Beam Interactions with Materials and Atoms, 234 (2005) 169-175.

[14] H.-M. Kuan, T.W. Bonner, J.R. Risser, An investigation of the $\mathrm{C} 12+\mathrm{He} 3$ reactions at bombarding energies between 1.8 and 5.4 MeV, Nuclear Physics, 51 (1964) 481-517.

[15] E.A. Wolicki, H.D. Holmgren, R.L. Johnston, E.G. Illsley, DIFFERENTIAL CROSS SECTIONS FOR THE BE-9(HE-3,P)B-11 REACTION, Physical Review, 116 (1959) 1585-1591.

[16] M. Mayer, SIMNRA User's Guide, in, Max-Planck-Institut für Plasmaphysik, Germany, Garching, Germany, 1997.

[17] U.v. Toussaint, unpublished.

[18] V. Philipps, T. Loarer, H.G. Esser, S. Vartanian, U. Kruezi, S. Brezinsek, G. Matthews, Dynamic fuel retention and release under ITER like wall conditions in JET, Journal of Nuclear Materials, 438 (2013) S1067-S1071. 
[19] M. Mayer, V. Rohde, A. Von Keudell, Characterisation of deposited hydrocarbon layers below the divertor and in the pumping ducts of ASDEX Upgrade, Journal of Nuclear Materials, 313-316 (2003) 429-433.

[20] J. Roth, C. Hopf, Sticking coefficient and surface loss probability of eroded species during bombardment of carbon with deuterium, Journal of Nuclear Materials, 334 (2004) 97-103. 
Figure captions

Figure 1: Positions of cavity samples in the JET divertor during a) the 1999-2001 and b) the 2005-2009 campaign. Strike point distributions in the same campaigns are shown at the bottom

Figure 2: Reconstructed incident particle flux distributions for the inner divertor cavity samples during the 1999-2001 and the 2005-2009 (red) campaigns. The reconstruction is based on the deposition of $\mathrm{D}$ on the bottom plate

Figure 3: Deposition inside the inner divertor cavities during a) the 1999-2001 and b) the 2005-2009 campaigns, experimental data (dots) and modeling (lines). Right scale is for D/C. The deposition on the bottom plate is shown in the lower part of the figure, the deposition on the inner top plates is shown in the upper part. Note that the axis for the top plates is from top to bottom

Figure 4: Reconstructed incident particle flux distribution for the septum cavity sample during the 1999-2001 campaign

Figure 5: Deposition inside the septum cavity during the 1999-2001 campaign, experimental data (dots) and modeling (lines). See Fig. 3 for details

Figure 6: Deposition inside the outer divertor cavity during 2005-2009 campaign, experimental data (dots) and modeling (lines). See Fig. 3 for details 

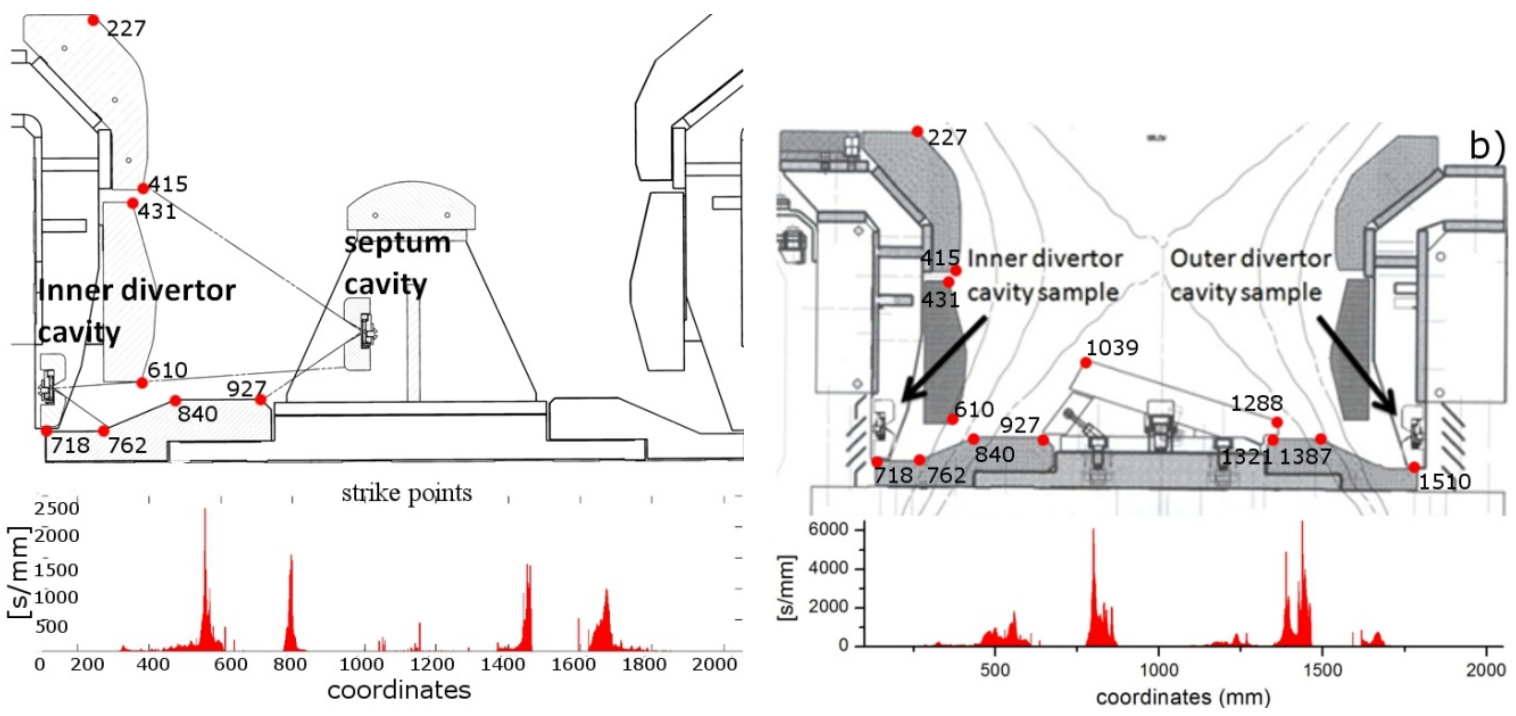

Figure 1 


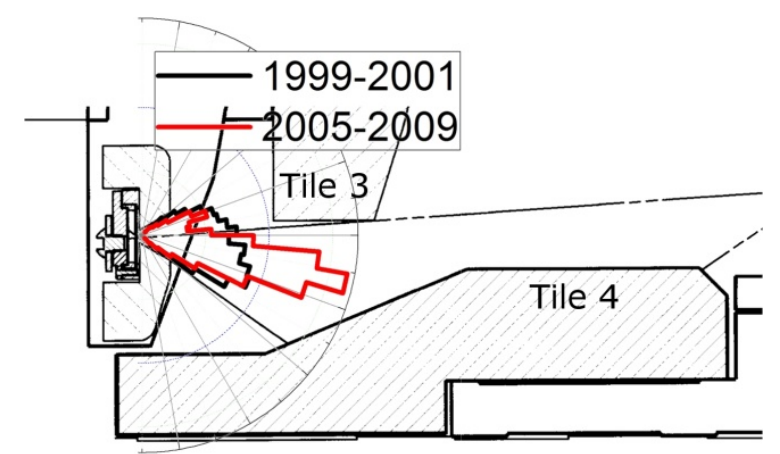

Figure 2 

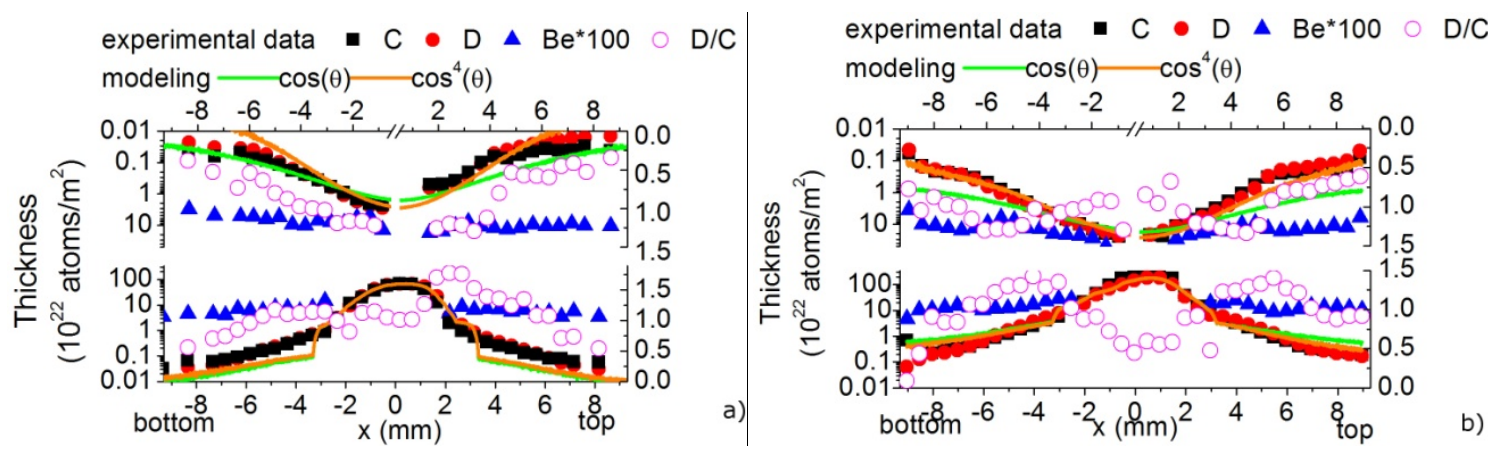

Figure 3 


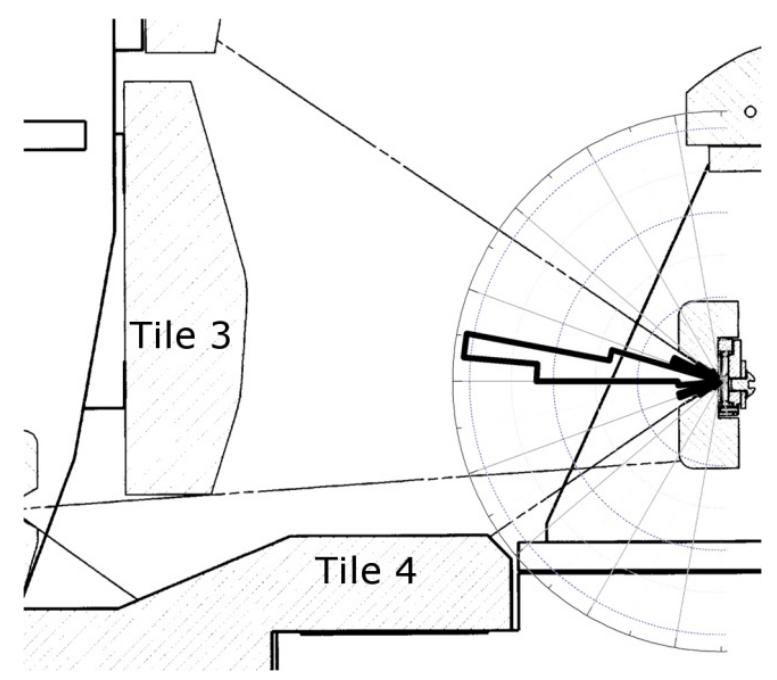

Figure 4 


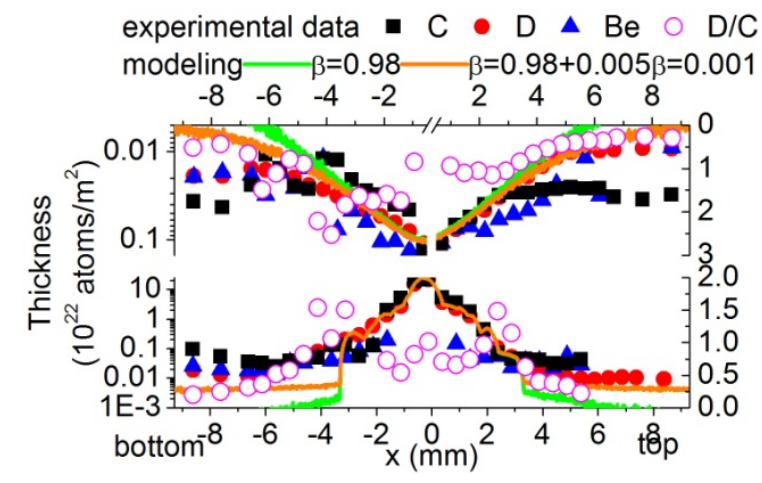

Figure 5 


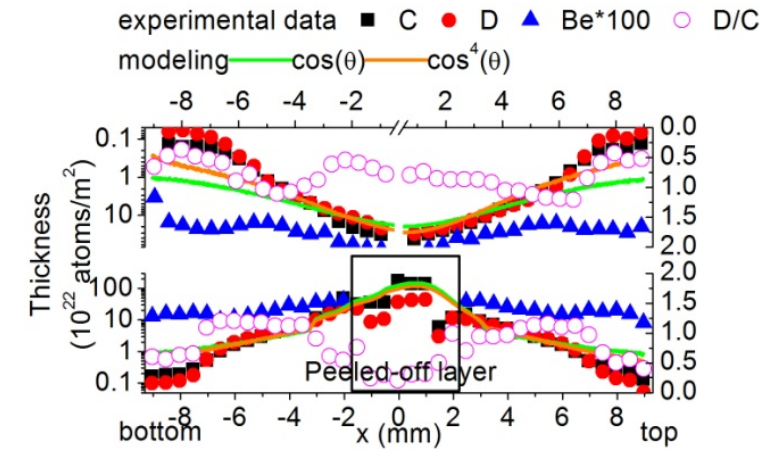

Figure 6 